Introduction/Background* Platinum-containing chemotherapy \pm bevacizumab is standard-of-care for recurrent/metastatic/ persistent $(\mathrm{R} / \mathrm{M} / \mathrm{P})$ cervical cancer (CC). Anti-PD-(L)1 therapy has benefit in some patients who progress after first-line (1L) therapy; 1L efficacy is unknown. HPV infection, implicated in $>95 \%$ of CCs, is linked to TGF- $\beta$ upregulation. Bintrafusp alfa is a first-in-class bifunctional fusion protein composed of the extracellular domain of the TGF- $\beta$ RII receptor (a TGF- $\beta$ 'trap') fused to a human IgG1 mAb blocking PDL1. Promising activity was observed in patients with recurrent, platinum-experienced CC (response rate 28.2\%). We report data from a phase $1 \mathrm{~b}$ trial evaluating safety of $1 \mathrm{~L}$ bintrafusp alfa + chemotherapy \pm bevacizumab (INTR@PID 046; NCT04551950).

Methodology Patients with R/M/P CC who had not received prior systemic therapy were eligible for cohort 1 . They received bintrafusp alfa $2400 \mathrm{mg}$ q3w plus cisplatin $50 \mathrm{mg} / \mathrm{m}^{2}$ or carboplatin AUC5, paclitaxel $175 \mathrm{mg} / \mathrm{m}^{2}$ with (cohort $1 \mathrm{~A}$ )/ without (cohort 1B) bevacizumab $15 \mathrm{mg} / \mathrm{kg}$ until disease progression, death, unacceptable toxicity, or withdrawal. Primary endpoints: occurrence of predefined dose-limiting toxicities $(D L T) \leq 4$ weeks from treatment start; adverse event occurrence. Target recruitment was 8 patients/cohort, with safety assessments when 3 and 8 patients had completed the DLT period.

Result(s)* As of May 4, 2021, 8 and 9 patients in cohorts 1A and $1 \mathrm{~B}$ had received therapy for a median of 10.6 and 9.0 weeks. All patients had completed the DLT period and remained on therapy. Two non-bintrafusp alfa-related DLTs were observed in cohort 1B (grade 4 amylase elevation, grade 3 menorrhagia); neither led to treatment discontinuation. Anygrade treatment-related adverse events (TRAEs) occurred in $62.5 \%$ and $100 \%$ of patients in cohorts $1 \mathrm{~A}$ and $1 \mathrm{~B}$. Grade 3 TRAEs occurred in 3 and 2 patients (cohort 1A: anemia $[\mathrm{n}=2]$, lipase increase, decreased neutrophil count, maculopapular rash $[\mathrm{n}=1$ each]; cohort $1 \mathrm{~B}$ : anemia, rectal hemorrhage, vaginal bleeding $[n=1$ each]); 1 patient in cohort $1 \mathrm{~B}$ had grade 4 anemia. No treatment-related deaths occurred. Preliminary efficacy based on short follow-up showed 3 and 2 tumor responses ( 2 and 1 pending confirmation) in cohorts $1 \mathrm{~A}$ and $1 \mathrm{~B}$.

Conclusion* No new safety signals were observed with $1 \mathrm{~L}$ bintrafusp alfa + chemotherapy \pm bevacizumab in patients with R/M/P CC. Further studies are warranted.

\section{ULTRASOUND ROLE IN STAGING OF CANCER CERVIX}

A Elagwany*. Alexandria university, Obg , Alexandria, Egypt

\subsection{6/ijgc-2021-ESG0.76}

Introduction/Background* Cancer cervix is common in developing countries due to limited adoption of screening programs . It is far less common in Developed countries due to availability of national screening program and governmental health insurance system . Cancer cervix in developing countries usually present in stage $1 \mathrm{~b}$ and beyond. MRI and EUA is usually used for staging before surgery .

Methodology Recently, due to advanced technologies in ultrasound, we can now stage cancer cervix accurately and replace MRI and EUA. We are trying here to spot the lights over this with a pictorial illustration of different stages. The accuracy of vaginal sonography for the evaluating cancer cervix is comparable to that of MRI and even better for local staging in identifying tissue planes

Result(s)* Ultrasound can be used in cancer cervix to assess the topography regarding exophytic versus endophytic tumor, The tumor size measured in three diameters and the distance between the tumor and the internal cervical os the pericervical fascia which is the paracervix at he level of the cervix and the paracolpos at the level of the vagina is assessed. Thence, the extent of the radical procedure (parametrectomy) can be planned.

The tumor is usually hypoechoic in cases of squamous cell carcinoma and hyperechoic in adencarcinoma. His is important in differentiating large bulky endocervical tumors (with regular outline) (stage 1) from those with paramretrial invasion with irregular outline (stage $2 \mathrm{~b}$ ). The vaginal extension is evaluated by the thickening or masses of the vaginal walls (stage 2a) along with assessing the paracolpos in the same manner as before (stage $2 \mathrm{~b}$ ). Ureteral dilatation is common in parametrial infiltration and is seen .

The spread into the urinary bladder and/or rectum (stage 4) can be determined and the ultrasound for the bladder involvement is better than does cystoscopy, as this can only show bullous mucosal edema or mucosal lesion but not the wall affection that can be seen by ultrasound. The assessment of both is based on assessing her muscle layer and the related fascia.

Conclusion* Ultrasound is comparable to mri in local staging of cancer cervix especially for minor changes .

\section{A LARGE, MULTICENTER, RETROSPECTIVE STUDY ON EFFICACY AND SAFETY OF STEREOTACTIC BODY RADIOTHERAPY IN OLIGOMETASTATIC CERVICAL CANCER (MITO-RT2/RAD)}

${ }^{1} \mathrm{G}$ Macchia, ${ }^{2} \mathrm{M}$ Campitelli, ${ }^{1} \mathrm{P}$ Bonome, ${ }^{3} \mathrm{C}$ Laliscia, ${ }^{4} \mathrm{~A}$ Fodor, ${ }^{5} \mathrm{~L}$ Draghini, ${ }^{6} \mathrm{P}$ Gentile, ${ }^{7} \mathrm{GR}$ D'agostino, ${ }^{8} \mathrm{~V}$ Balcet, ${ }^{9} \mathrm{~A}$ Raguso, ${ }^{10} \mathrm{E}$ Ippolito, ${ }^{11 ; 12} \mathrm{M}$ Ferioli, ${ }^{13} \mathrm{~L}$ Vicenzi, ${ }^{14} \mathrm{~S}$ Borghesi, ${ }^{2} \mathrm{P}$ Mitidieri, ${ }^{15} \mathrm{VDI}$ Cataldo, ${ }^{16} \mathrm{E}$ Perrucci, ${ }^{17} \mathrm{~S}$ Pignata, ${ }^{18} \mathrm{G}$ Scambia, ${ }^{19} \mathrm{G}$ Ferrandina* ${ }^{1}$ Gemelli Molise Hospital - Università Cattolica del Sacro Cuore, Radiation Oncology Unit, Campobasso, Italy; ${ }^{2}$ Fondazione Policlinico Universitario A. Gemelli, IRCCS, UOC di Radioterapia, Dipartimento di Scienze Radiologiche, Radioterapiche ed Ematologiche, Rome, Italy; ${ }^{3}$ University of Pisa, Department of Translational Medicine, Division of Radiation Oncology, Pisa, Italy; ${ }^{4}$ IRCCS San Raffaele Scientific Institute, Department of Radiation Oncology, Milan, Italy; ${ }^{5}$ S. Maria Hospital, Radiation Oncology Centre, Terni, Italy; ${ }^{6}$ UPMC Hillman Cancer Center San Pietro FBF, Radiation Oncology Unit, Rome, Italy; 'Humanitas Clinical and Research Hospital, IRCSS, Department of Radiotherapy and Radiosurgery, Rozzano, Italy; ${ }^{8}$ Nuovo Ospedale degli Infermi, UOC Radioterapia, Biella, Italy; ${ }^{9}$ Fondazione 'Casa Sollievo della Sofferenza', IRCCS, UOC Radioterapia, S. Giovanni Rotondo (FG); ${ }^{10}$ Campus Bio-Medico University, Department of Radiation Oncology, Rome, Italy; ${ }^{11}$ Radiation Oncology, IRCCS Azienda Ospedaliero-Universitaria di Bologna, Bologna; ${ }^{12}$ Department of Experimental, Diagnostic and Specialty Medicine - DIMES, Alma Mater Studiorum University of Bologna; ${ }^{13}$ Azienda Ospedaliera Universitaria Ospedali Riuniti, Radiation Oncology Unit, , Ancona, Italy; ${ }^{14}$ Azienda USL Toscana Sud Est, Radiation Oncology Unit of Arezzo-Valdarno, Arezzo, Italy; ${ }^{15}$ University of Florence, Radiation Oncology Unit, Oncology Department, Firenze, Italy; ${ }^{16}$ Perugia General Hospital, Italy, Radiation Oncology Section, Perugia, Italy; ${ }^{17}$ Istituto Nazionale Tumori di Napoli, Fondazione Pascale IRCCS, Naples, Italy; ${ }^{18}$ Fondazione Policlinico Universitario A. Gemelli, IRCCS, UOC Ginecologia Oncologica, Dipartimento per la salute della Donna e del Bambino e della Salute Pubblica, Roma, Italy; ${ }^{19}$ Fondazione Policlinico Universitario A. Gemelli, IRCCS, UOC Ginecologia Oncologica, Dipartimento per la salute della Donna e del Bambino e della Salute Pubblica, Roma, Italy

\subsection{6/ijgc-2021-ESG0.77}

Introduction/Background* Data supporting stereotactic body radiotherapy (SBRT) for oligometastatic gynecological cancer patients are increasing, but stereotactic treatments have not 